\title{
Bioinformatic Analysis of Strawberry PGR5 Gene
}

\author{
Xiran Wang, Jiangang He, Haoru Tang \\ Sichuan Agriculture University, Chengdu, China.
}

Keywords: PGR5; Morus notabiliswere closest relative; bioinformation analyses.

\begin{abstract}
PGR5 has always been known as a key factor of Heat response in plant testa. Recent studies show that PGR5 (proton gradentregulation 5) is a proton gradient regulatory protein, which transfers cycle electronic I encoding thylakoid membrane proteins and optical systems, and helps to induce $\mathrm{pH}$ heat dissipation. In this research we chosen PGR5 gene of 9 kinds species, downloaded their nucleotide and protein sequence from NCBI as the research object, found strawberry PGR5 gene via bioinformation analyses, constructed phylogenetic tree. At the same time, we analyzed the strawberry PGR5 gene of physical and chemical properties and its protein structure and so on. The phylogenetic tree showed that strawberry and Morus notabiliswere closest relative. By the protein prediction, we found that the protein owed one proper signal peptide without obvious transmembrane regions.
\end{abstract}

\section{Introduction}

PGR5 (proton gradentregulation 5) is a proton gradient regulatory protein, which transfers cycle electronic I encoding thylakoid membrane proteins and optical systems, and helps to induce $\mathrm{pH}$ heat dissipation[1]. Recent studies show that In photosynthesis, PSI ring electron transfer consists of two pathways, one requiring a complex compound protein known as NADH or NDH dehydrogenase complex [2] and the other one of at least two proteins: PGR5 complex [3] proton gradient regulated protein 5 (proton gradient regulation 5, PGR5 1 (PGR5-like) and protein protein 1 (A, PGRL1) located in the thylakoid membrane protein), like protein 1 and PGR5 interaction in structure and function. PGR5 and PGRL1 interact with PSI and form a complex, causing PsbS to control qE (quantum electronics) [4]. The $\mathrm{qE}$ mechanism of light protection is stimulated under the low $\mathrm{pH}$ cavity, and the PGR5 protein is used to acidification the cavities of the cavities of [5]. The PGRL1-PGR5 complex participates in the transformation of residual electron flow to CEF, accepts electron from Fd (feroxin) and restores PQ (plastid quinone) library. Therefore, it acts as a Fd-PQ reductase [6] to promote the cyclic electron flow cycle. Light is the root cause of PSI photoperiod, and the ring type electron transfer can be up-regulated at the time of drought stimulation. Linear electron transfer 3 times more than; in the long term under high light stress, Arabidopsis Fd2-KO plant plastoquinone pool by cyclic electron transfer to PGR5 improvement depends on the relative balance of reduction oxidation recovery level, fd2 and pgr5 in high light photosynthesis severely damaged, Fd2-KO plants showed that PGR5, [29] play a role in adaptation light on the high strength of the. The cyclic electron transfer dependent on proton gradient regulatory protein (proton gradentregulation 5, PGR5) contributes to the redox balance of chloroplasts. When plants are destroyed, such as rice chloroplasts, PGR5 is the [7] necessary to maintain the ability of redox protein quinone reduction.

\section{Result and Analysis}

\subsection{Strawberry PGR5 Find and Phylogenetic Tree Construction of PGR5}

Run BLAST in the Fragaria ×ananassa database of GDR and Strawberry GARDEN respectively using Arabidopsis thaliana PGR5 as a sample. Select the sequence with ideal S and C value, we find strawberry PGR5.

We have chosen PGR5-like gene of 9 kinds species (Table 1), downloaded their nucleotide and protein sequence from NCBI as the research object, analyzed sequences via MEGA6.0, constructed 
phylogenetic tree on the genetic distance base (Figure 1). The relationship between strawberry and petunia is closer than others, due to they all contain a lot of anthocyanins probably.

Table 1. Basic information of pgr5 gene in different species

\begin{tabular}{|cccc|}
\hline Species & mRNA Accession Number & Protein Accession Number & $\begin{array}{c}\text { Amino Acid } \\
\text { Number }\end{array}$ \\
\hline Fragaria vesca & XM_004296356.2 & 764568155 & 880 \\
Morus notabilis & XM_010111443.1 & 703149999 & 372 \\
Cucumis sativus & XM_004133996.2 & 778678100 & 855 \\
Cucumis melo & XM_008440250.2 & 1035393427 & 942 \\
Gossypium arboreum & XM_017759347.1 & 1050587011 & 550 \\
Gossypium hirsutum & XM_016821285.1 & 1029025831 & 606 \\
Citrus clementina & XM_006420254.1 & 567854394 & 930 \\
Citrus sinensis & XM_006489596.1 & 568873020 & 758 \\
$\begin{array}{c}\text { Cucumis melo genomic } \\
\text { chromosome }\end{array}$ & LN713260 & DHL92 & 35939859 \\
\hline
\end{tabular}

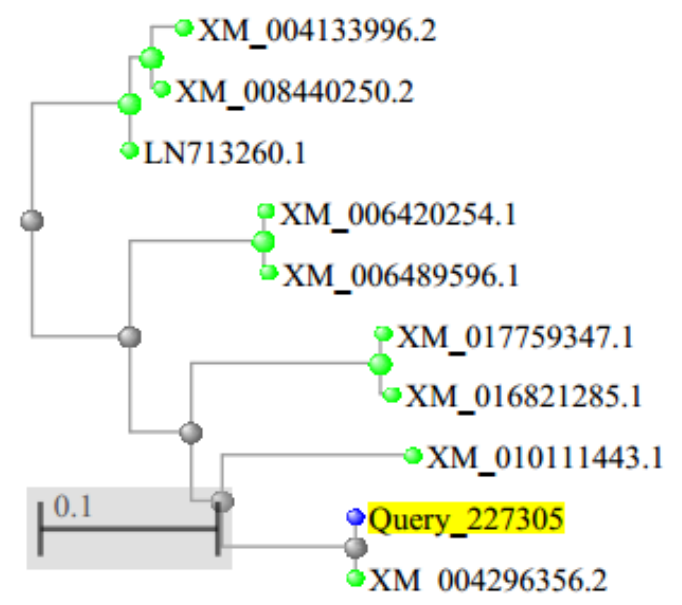

Figure 1. Phylogenetic Tree of Strawberry PGR5.

\subsection{Strawberry PGR5 Protein Physical Analysis}

Analyze strawberry PGR5 protein physical feature using ProtPram. Result shows as following: the protein is composed by $381 \mathrm{bp}$, molecular weight is 30558.94 , theoretical $\mathrm{pI}$ is 5.26 , electric neutrality, molecular formula isC1127H1875N381O466S73. Because the $\mathrm{N}$-terminal of the sequence considered is A (Ala), the estimated half-life is 30 hours in mammalian reticulocytes (in vitro), 20 hours in yeast (in vitro) and 10 hours in Escherichia coli (in vitro). The instability index (II) is computed to be 34.88 , so the protein is unstable. The aliphatic index of the protein is 32.55 . Grand average of hydropathicity (GRAVY): 0.806.

Amino acid composition result is settings as following (Table 2): the peptide chain consists many Ala as 32.5\%.

Table 2. Strawberry pgr5 gene amino acid composition

\begin{tabular}{|ccc|}
\hline Amino Acid & Count & Percentage \\
\hline Ala (A) & 124 & $32.5 \%$ \\
Cys (C) & 73 & $19.2 \%$ \\
Gly (G) & 100 & $26.2 \%$ \\
Thr (T) & 84 & $22.0 \%$ \\
\hline
\end{tabular}

2.3 Strawberry PGR5 Protein Signal Peptide Forecast

Forecast strawberry PGR5 protein signal peptide using SignalP 4.1. Result shows as following (Figure 2). There is one signal peptide in the PGR5 between 19th base and 20th base. 


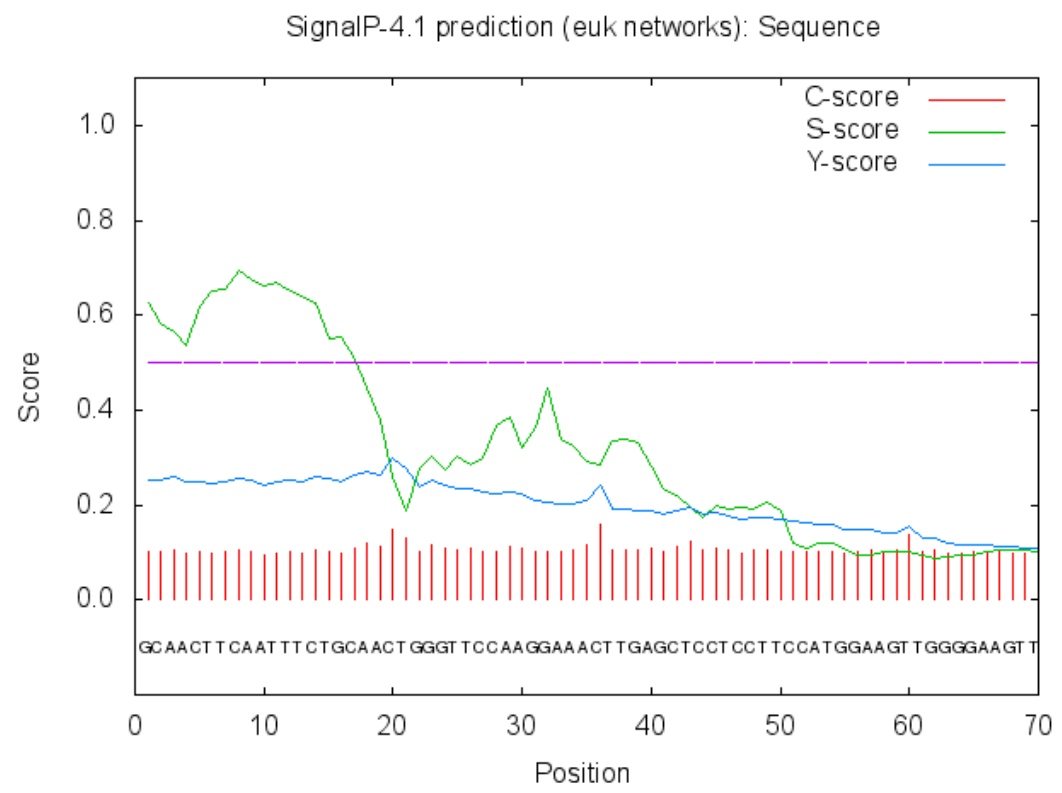

Figure 2. Signal peptide forecast of strawberry PGR5.

\subsection{Strawberry PGR5 Protein Transmembrane Domain Forecast}

Forecast strawberry PGR5 protein transmembrane domain using TMHMM Server. Result shows as following (Figure 2). There isn't transmembrane domain in the strawberry PGR5, thus the protein is not cytomembrane receptor or located on cytomembrane.

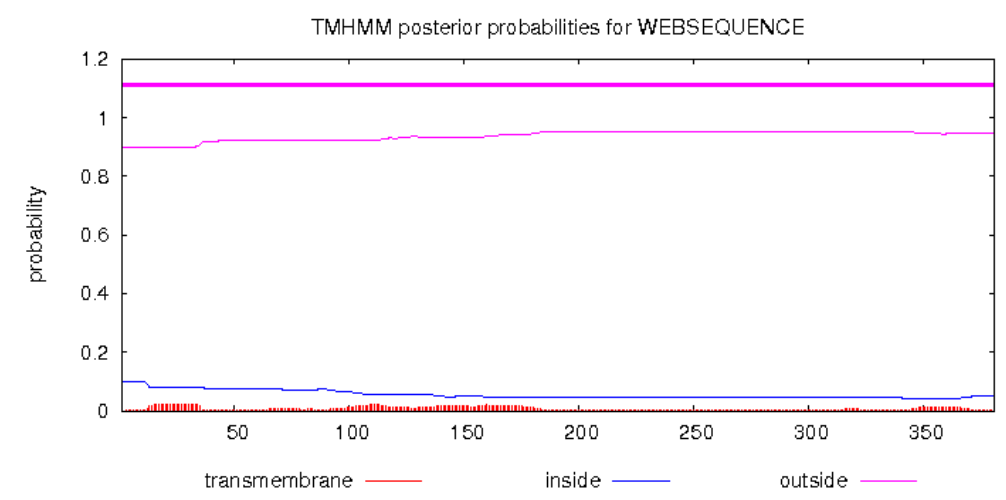

Figure 3. Transmembrane domain forecast of strawberry PGR5.

\section{Materials and Methods}

Sequences access from NCBI (https://www.ncbi.nlm.nih.gov/). BLAST run in GDR (https://www.rosaceae.org/), and Strawberry GARDEN(http://strawberry-garden.kazusa.or.jp/). Phylogenetic tree is constructed via MEGA6.0, forecast strawberry PGR5 protein signal peptide using SignalP 4.1 (http://www.cbs.dtu.dk/services/SignalP/), forecast strawberry PGR5 protein transmembrane domain using TMHMM Server (http://www.cbs.dtu.dk/services/TMHMM/).

\section{Conclusion}

Enhanced hydrogen production rates in the pgrl1, pgr5, and the pgr5 pgrl1 double mutant under S deplete conditions lead to the highest continuous photobiological produced hydrogen amounts of eukaryotic cells reported so far. These rates are achieved by a prolonged residual PSII activity providing an increased electron supply toward the hydrogenase. PSII activity can be maintained in the mutants without inhibiting the oxygen sensitive hydrogenase, because the oxygen consumption capacity is increased. Our results suggest that respiration and light-dependent O2-uptake rates are higher in the pgr mutants, and that this is responsible for the faster transition to anaerobiosis, 
especially when the greater residual PSII activity of the mutants is taken into account [8]. The exploration on strawberry TT12, which is forward gene to strawberry PGR5, have been attempt in our lab, we would focus on the interaction between strawberry TT12 and PGR5 so as to provide reference for further study and utilization.

\section{Acknowledgments}

I thank my teachers: Haoru Tang, for the directions, Jiangang He for the discussion. This work was financially supported by National Innovation Experiment Program for University Students (201510626005) fund.

\section{References}

[1]. Huang W, Yang S-J, Zhang S-B, Zhang J-L, Cao K-F. Cyclic electron flow plays an important role in photoprotection for the resurrection plant Paraboea rufescens under drought stress.Planta, 2012, 430: 288-292.

[2]. Hertle, A P, Blunder T, Wunder T, Pesaresi P, Pribil M, Armbruster U,and Leister D. PGRL 1 is the elusive ferredoxin-plastoquinone reductase in photosynthetic cyclic electron flow. Mol.Cell, 2013, 49: 511-523.

[3]. Nishikawa Y, Yamamoto H, Okegawa Y, Wada S, Sato N,Taira Y, Sugimoto K, Makino A, Shikanai T. PGR5-dependent cyclic electron transport around PSI contributesto the redox homeostasis in chloroplasts rather than $\mathrm{CO} 2$ fixation and biomass production in rice. Plant and Cell Physiology, 2012, 53: 2117-2126.

[4]. Peng L, Yamamoto, $\mathrm{H}$ and SHlkanai T. Structure and biogenesis of the chloropiast NAD (P) H dehydrogenase complex. Biochim. Blophys.Acta, 2011, 1807: 945-953.

[5]. Nellaepalli S, Kodru S, Raghavendra A S, et al. Antimycin a sensitive pathway independent from PGR5 cyclic electron transfer triggers non-photochemical reduction of PQ pool and state transition in Arabidopsis thaliana [J].J Photochem Photobiol B,2015,146: 24-33

[6]. Steinbeck J, Nikolova D, Weingarten R, et al. Deletion of Proton Gradient Regulation 5 (PGR5) and PGR5-Like 1 (PGRL1) proteins promote sustainable light-driven hydrogen production in Chlamydomonas reinhardtii due to increased PSII activity under sulfur deprivation. [J]. Frontiers in Plant Science, 2015, 6.77-83.

[7]. Kou J, Takahashi S, Fan D Y, et al. Partially dissecting the steady-state electron fluxes in Photosystem I in wild-type and pgr5 and ndh mutants of Arabidopsis[J]. Frontiers in Plant Science, 2015, 6(758):758-776.

[8]. Steinbeck J, Nikolova D, Weingarten R, et al. Deletion of Proton Gradient Regulation 5 (PGR5) and PGR5-Like 1 (PGRL1) proteins promote sustainable light-driven hydrogen production in Chlamydomonas reinhardtii due to increased PSII activity under sulfur deprivation.[J]. Frontiers in Plant Science, 2015, 6.89-93. 